\title{
The rare and forbidden: testing physics beyond the Standard Model with Mu3e
}

\author{
Ann Kathrin Perrevoort ${ }^{1 \star}$ on behalf of the Mu3e Collaboration ${ }^{2}$ \\ 1 NIKHEF, Amsterdam, Netherlands (formerly Physics Institute, Heidelberg, Germany) \\ 2 Paul Scherrer Institute, Villigen, Switzerland \\ $\star$ ann-kathrin.perrevoort@cern.ch
}

Proceedings for the 15th International Workshop on Tau Lepton Physics, Amsterdam, The Netherlands, 24-28 September 2018

doi:10.21468/SciPostPhysProc. 1

\begin{abstract}
The upcoming Mu3e experiment aims to search for the lepton flavour violating decay $\mu^{+} \rightarrow e^{+} e^{-} e^{+}$with an unprecedented final sensitivity of one signal decay in $10^{16} \mathrm{ob}-$ served muon decays by making use of an innovative experimental design based on novel ultra-thin silicon pixel sensors. In a first phase, the experiment is operated at an existing muon beam line with rates of up to $10^{8}$ muons per second. Detailed simulation studies confirm the feasibility of background-free operation and project single event sensitivities in the order of $10^{-15}$ for signal decays modelled in an effective field theory approach. The precise tracking of the decay electrons and large geometric and momentum acceptance of Mu3e enable searches for physics beyond the Standard Model in further signatures. Examples of which are searches for lepton flavour violating two-body decays of the muon into an electron and an undetected boson as well as for electron-positron resonances in $\mu^{+} \rightarrow e^{+} e^{-} e^{+} \bar{v}_{\mu} v_{e}$ which could result for instance from a dark photon decay. The Mu3e experiment is expected to be competitive in all of these channels already in phase I.
\end{abstract}

(c) (1) Copyright A. K. Perrevoort et al. This work is licensed under the Creative Commons Attribution 4.0 International License.

Published by the SciPost Foundation.
Received 16-11-2018

Accepted 17-01-2019

Published 22-02-2019

doi:10.21468/SciPostPhysProc.1.052

\section{Contents}

1 Introduction $\quad 2$

2 The Mu3e Experiment $\quad 2$

2.1 Signal and Background 2

2.2 Experimental Concept 3

3 Sensitivity Studies $\quad \mathbf{5}$

$3.1 \mu \rightarrow$ eee in Effective Theories $r$

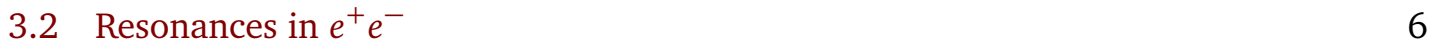




\section{Introduction}

The flavour of leptons is conserved in the Standard Model but - as demonstrated by the observation of neutrino oscillations - it is not conserved in nature. The violation of the flavour of charged leptons has however eluded observation so far.

One example for charged lepton flavour violation is the decay $\mu^{+} \rightarrow e^{+} e^{-} e^{+}$. In a Standard Model extended to include neutrino mixing, it can be mediated in loop diagrams (see figure 1a) but it is suppressed to branching fractions below $10^{-54}$ and thus far beyond what experiments can observe. Any observation of $\mu \rightarrow$ eee would therefore be a clear sign for physics beyond the Standard Model. Indeed, many extensions of the Standard Model predict enhanced rates for $\mu \rightarrow$ eee, for example via loop diagrams with supersymmetric particles (see figure $1 \mathrm{~b}$ ) or at tree-level via a $Z^{\prime}$ (see figure 1c).

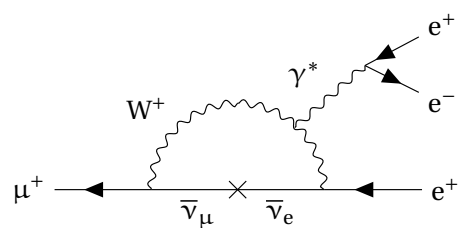

(a) Neutrino mixing.

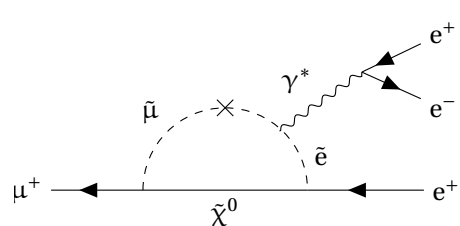

(b) Supersymmetry.

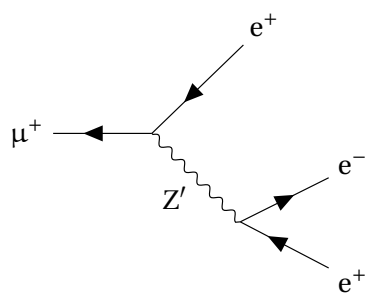

(c) Extended electroweak sector.

Figure 1: The decay $\mu \rightarrow$ eee via neutrino mixing and in other extensions of the Standard Model.

\section{The Mu3e Experiment}

The latest measurement of $\mu^{+} \rightarrow e^{+} e^{-} e^{+}$has been performed by the SINDRUM experiment [1]. As no signal was observed, branching fractions of larger $1.0 \times 10^{-12}$ were excluded at $90 \%$ confidence limit (CL). The upcoming Mu3e experiment at the Paul Scherrer Institute (PSI) will repeat this search with a sensitivity of about one signal decay in $10^{15}$ muon decays in the first phase of the experiment, and ultimately one signal decay in $10^{16}$ muon decays in the second phase, thus improving the existing limit by four orders of magnitude [2].

\subsection{Signal and Background}

The signal decay $\mu^{+} \rightarrow e^{+} e^{-} e^{+}$is characterised by the coincidence of two positrons and one electron ${ }^{1}$ from a common vertex. As muons are stopped in the detector and decay at rest, the

\footnotetext{
${ }^{1}$ In the following, the two positrons and the electron will simply be referred to as three electrons.
} 


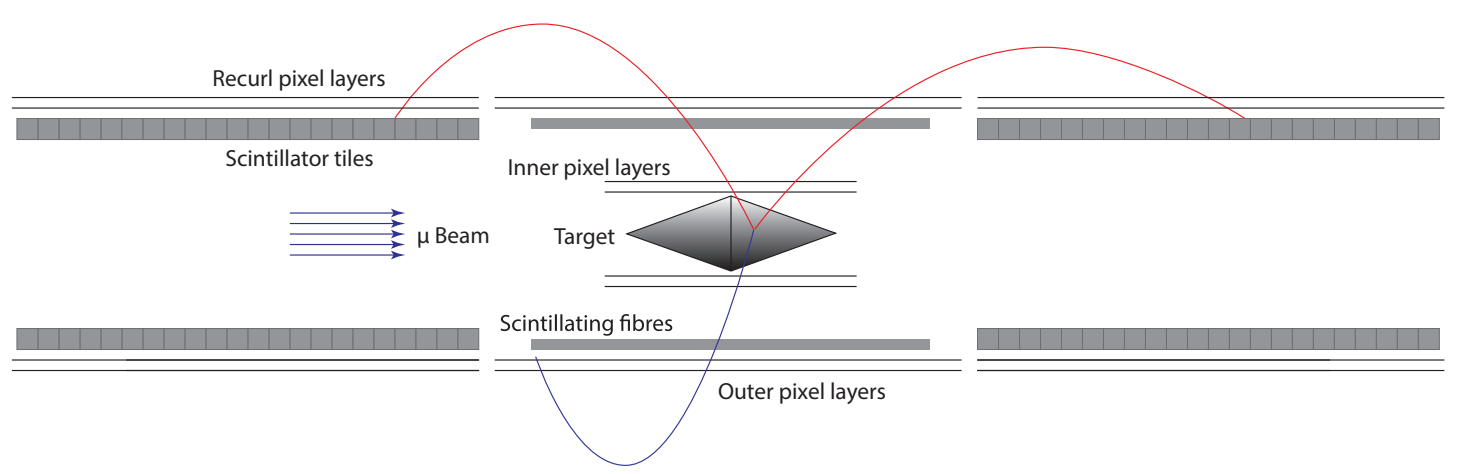

Figure 2: Schematic of the phase I Mu3e experiment in lateral projection with a simulated $\mu \rightarrow$ eee event.

momenta of the three decay products vanishes whereas the invariant mass equals the muon rest mass.

There are two sources of background to $\mu \rightarrow$ eee searches. On the one hand, there is background from the rare muon decay $\mu^{+} \rightarrow e^{+} e^{-} e^{+} \bar{v}_{\mu} v_{e}$ which has the same visible final state as $\mu^{+} \rightarrow e^{+} e^{-} e^{+}$. This source of background can be distinguished from signal by measuring the missing energy carried by the undetected neutrinos.

On the other hand, high muon stopping rates give rise to accidental combinations of two positrons and an electron from different processes. The most common source of this background stems from positrons that undergo Bhabha scattering in the detector material and have a considerable momentum transfer to the electron. Paired with another positron from a close-by Michel decay, these three particles can mimic a signal decay. In addition to kinematic constraints, this type of background is suppressed by means of vertexing and timing.

Thus, for the Mu3e experiment a very accurate electron tracking and a precise timing measurement are required in addition to high muon stopping rates.

\subsection{Experimental Concept}

The Mu3e experiment is designed to measure low momentum electrons with outmost precision and at high rates. In phase I, it will be located at an existing muon beam line at PSI which can provide muon rates of about $10^{8} \mu / \mathrm{s}$. In the second phase, higher muon rates in the range of $10^{9} \mu / \mathrm{s}$ are required. PSI is currently investigating on options for the High Intensity Muon Beam line with muon rates of the order of $10^{10} \mu / \mathrm{s}$.

The detector is shown in figure 2. The incoming muon beam is stopped on a hollow double cone target made from Mylar foil. The momentum of the decay products is measured by their curvature in the solenoidal magnetic field. The tracking detector is based on silicon pixel sensors and is cylindrically arranged around the beam axis. Two layers of pixel sensors surround the target for precise vertexing while two further layers are placed at a larger radius for momentum measurements. A minimum transverse momentum $p_{\mathrm{T}}$ of $10 \mathrm{MeV}$ is required for a particle to cross all four layers and be reconstructed. A scintillating fibre detector provides precise timing.

The electrons from muon decays have low momenta of only a few tens of MeV. Hence, multiple Coulomb scattering dominates the momentum resolution. For this reason, the HV-MAPS (High Voltage Monolithic Pixel Sensor) technology is chosen for the pixel detector [3]. This technology features a built-in readout circuitry eliminating the need for additional readout chips. HV-MAPS further allow for a sensor thickness of only $50 \mu \mathrm{m}$ and thus a material amount of $0.1 \%$ of a radiation length per pixel layer including mechanical support and readout flexprint. The precision of the momentum measurement can be further improved by measuring the tra- 

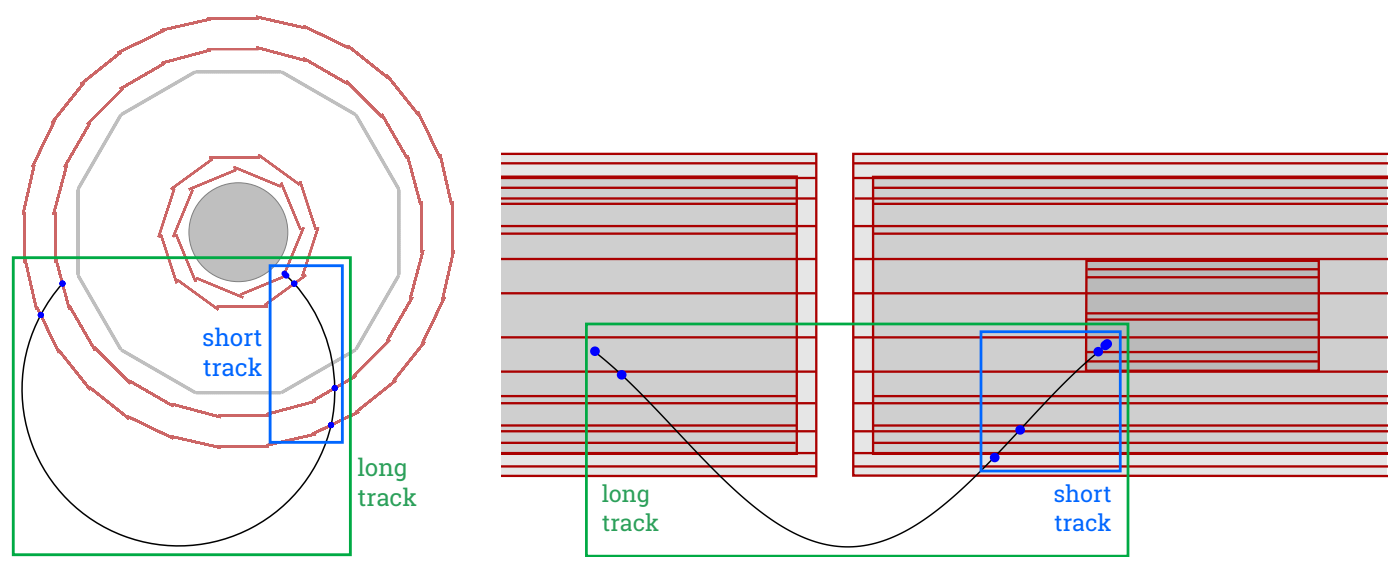

Figure 3: Event display of a simulated recurling track shown in transverse and longitudinal projection. The definitions of a reconstructed short and long track are shown.

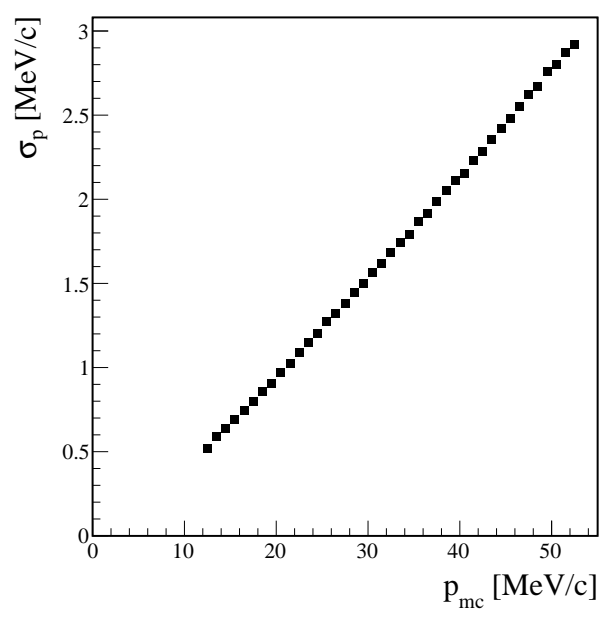

(a) Short tracks.

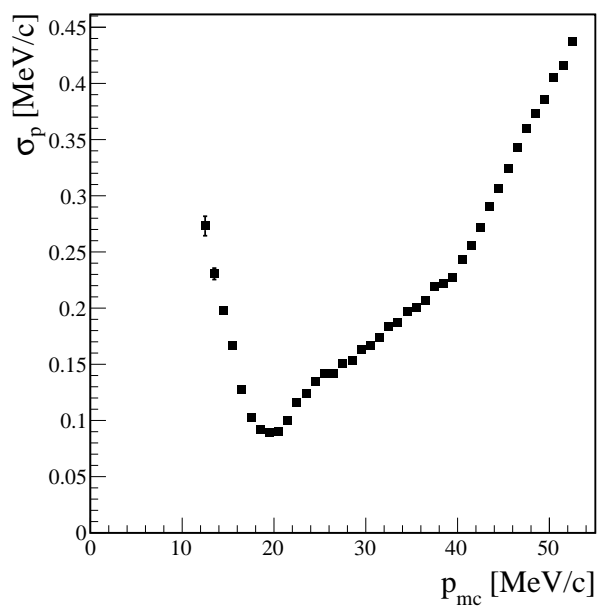

(b) Long tracks.

Figure 4: Momentum resolution of reconstructed short and long tracks.

jectory of the electron not only when it is outgoing but also when it returns to the detector (see figure 3). The momentum resolution of a reconstructed long track from a recurling particle is up to factor of ten smaller than in the case of reconstructed short tracks from the outgoing trajectory alone (see figure 4). The Mu3e detector is therefore extended by one (two) so-called recurl stations upstream and downstream of the central detector in phase I (phase II). The recurl stations consist of two layers of pixel sensors and scintillating tiles.

The data acquisition is performed without a hardware trigger. All sub-detectors are continuously read out. The data rate is however too high for mass storage so that $\mu \rightarrow$ eee candidates already need to be identified online. This is performed on the online filter farm which is based on graphics processing units. First, short tracks in the central detector are reconstructed in a fast triplet fit [4]. In the following vertex fit, $\mu \rightarrow$ eee candidates are looked for and if found all data of the corresponding readout frame is written to mass storage. Reconstruction of long tracks and refined vertex fits are then performed offline. 


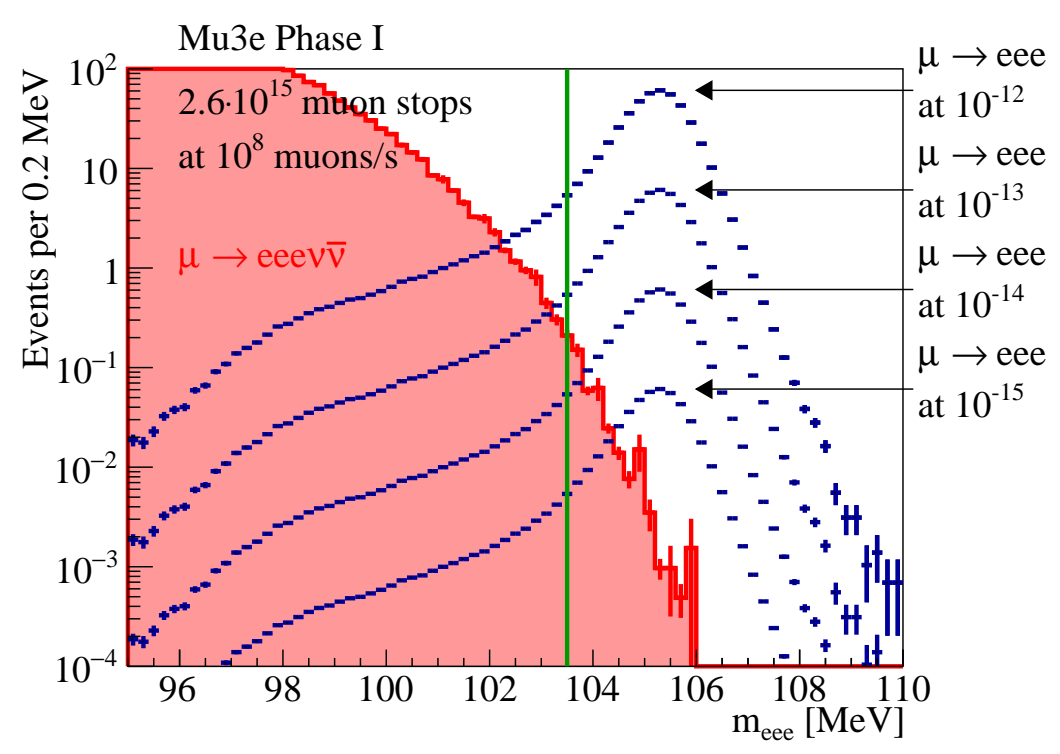

Figure 5: Expected distribution of the reconstructed invariant mass of $\mu \rightarrow$ eee candidates. Background from $\mu \rightarrow$ eee $v v$ decays is shown in red. The signal distribution (blue) is given for various assumptions on the $\mu \rightarrow$ eee branching fraction. The applied cut on the three electron mass is indicated by the green line.

\section{Sensitivity Studies}

The following sensitivity studies are performed with a detailed GEANT4-based simulation of the Mu3e detector in phase I. The muon stopping rate is assumed to be $10^{8} \mu / \mathrm{s}$, and the total run time is estimated with 300 data taking days which accumulates to a total of $2.6 \times 10^{15}$ muon decays.

\section{1 $\mu \rightarrow$ eee in Effective Theories}

In a baseline approach, the $\mu \rightarrow$ eee signal decay is implemented as a three-body decay without any assumptions on the underlying physics. In the event selection, only vertices with three reconstructed long tracks are kept because the momentum resolution of short tracks does not suffice to suppress background from $\mu \rightarrow$ eee $v \nu$. Constraints are applied on the quality of the vertex fit, the distance of the reconstructed vertex to the target, the relative timing between the electron candidates, as well as on the total momentum and invariant mass of the $\mu \rightarrow$ eee candidates. Figure 5 shows the expected distribution of the invariant three electron mass for background from $\mu \rightarrow$ eee $v v$ as well as for the $\mu \rightarrow$ eee signal at various branching fractions. With an expected number of 0.44 background events, background-free operation is confirmed. The phase I experiment is estimated to be sensitive to branching fractions as small as $5.2 \times 10^{-15}$ at $90 \% \mathrm{CL}$.

This approach however neglects the fact that the type of interaction which mediates $\mu \rightarrow$ eee affects the kinematics of the decay and consequently the efficiency to reconstruct signal events. The signal decay is therefore modelled with an effective field theory approach of dimension six. The operator basis and differential branching fraction are taken from $[5,6]$.

In figure 6 , Dalitz plots of $e^{+} e^{-}$pairs in $\mu \rightarrow$ eee are shown for three-body decays and for various effective operators. The generated three-body decays are evenly distributed over the accessible phase space as expected. The Dalitz plot of the reconstructed three-body decays 


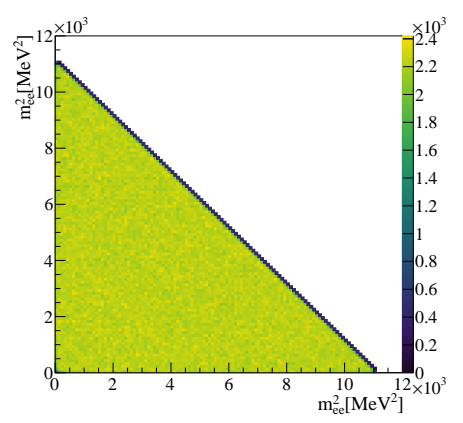

(a) Generated $\mu \rightarrow$ eee decays.

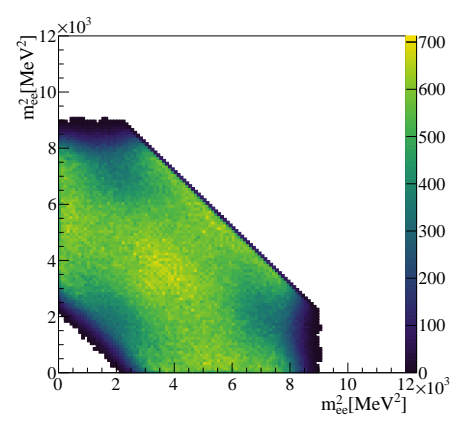

(b) Reconstructed three-body $\mu \rightarrow$ eee decays.

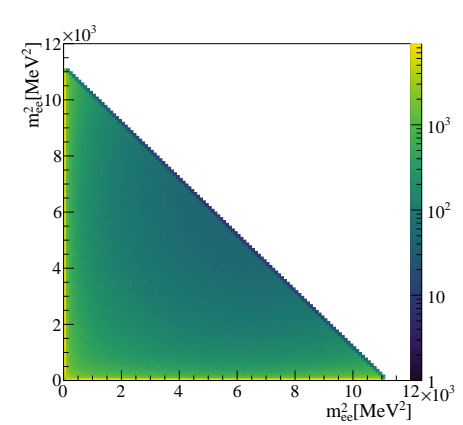

(c) Dipole operator. Note the logarithmic scale.

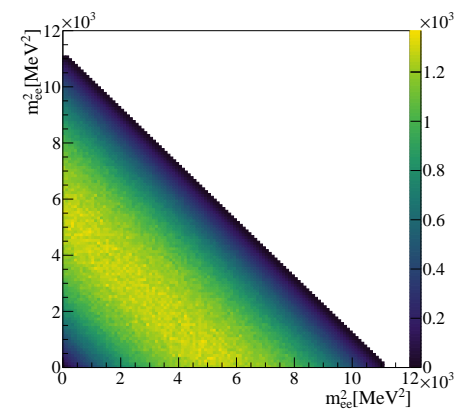

(d) Scalar 4-fermion operator.

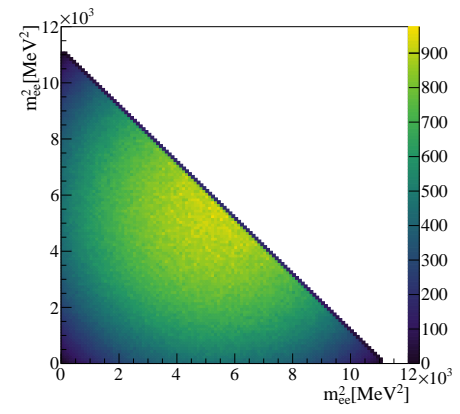

(e) Vector 4-fermion operator.

Figure 6: Dalitz plots of $e^{+} e^{-}$pairs in simulated $\mu \rightarrow$ eee decays. In the effective theory approach, the operators are evaluated individually.

illustrates the acceptance of the detector. The corners are cut as a result of the minimum detectable $p_{\mathrm{T}}$. Further structure stems from requiring long tracks only.

In the case of dipole operators, the virtual photon manifests in very small invariant $e^{+} e^{-}$ masses which increases the probability to have at least one decay particle with $p_{\mathrm{T}}$ below the detection threshold. Thus, the sensitivity is reduced compared to three-body decays with $\mathrm{BR} \geq 8.5 \times 10^{-15}$ at $90 \% \mathrm{CL}$. In the case of4-fermion operators on the contrary, the decay energy tends to be more equally distributed amongst the decay particles which leads to an increased detection efficiency. For all 4-fermion operators, comparable sensitivities of $\mathrm{BR} \geq 4.6 \times 10^{-15}$ at $90 \% \mathrm{CL}$ are estimated despite the distinct decay distributions.

This effective operator approach further provides a base for the interpretation of observations or non-observations in the various lepton flavour violation searches. A unique feature of $\mu \rightarrow$ eee searches is that in case of a discovery conclusions on the type of interaction can already been drawn from decay distributions such as Dalitz plots and angular distributions.

\subsection{Resonances in $e^{+} e^{-}$}

In the search for $\mu \rightarrow$ eee events, also a unprecedented number of $\mu \rightarrow$ eee $\nu v$ decays will be collected. As no constraint on the invariant three electron mass is applied in online reconstruction and event filtering, all $\mu \rightarrow$ eee $v v$ decays in the acceptance are recorded. This data can be used for resonance searches in $e^{+} e^{-}$pairs.

These resonances can be the signature of a dark photon - a potential messenger to a dark sector. The dark photon interacts via kinetic mixing with the Standard Model photon and $Z$ and thus couples to the electro-magnetic current. If sufficiently light, it can be radiated in muon 


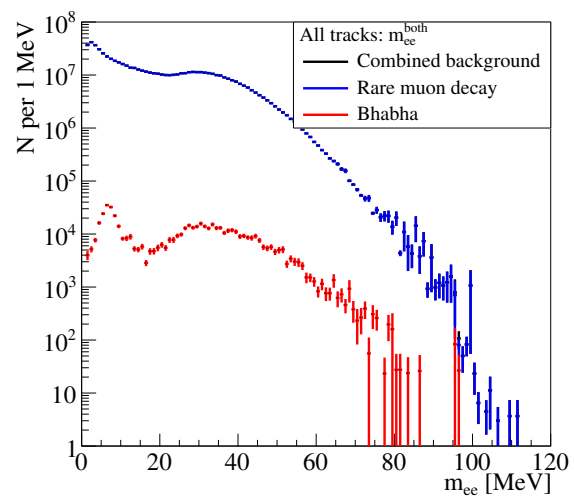

(a) Simulated background events. Both $e^{+} e^{-}$ combinations are considered.

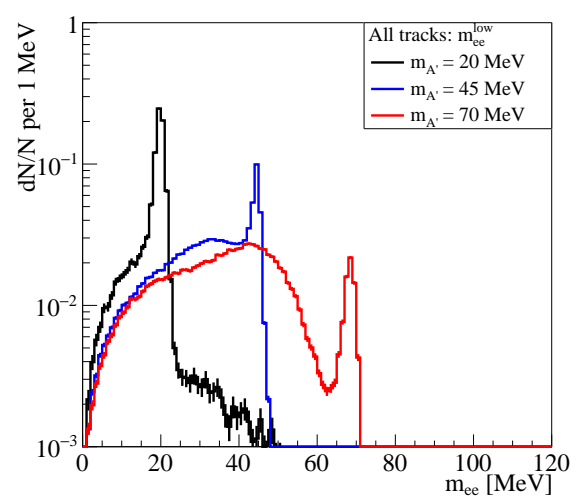

(c) Simulated dark photon signal events. The $e^{+} e^{-}$combination with the lower invariant mass is shown.

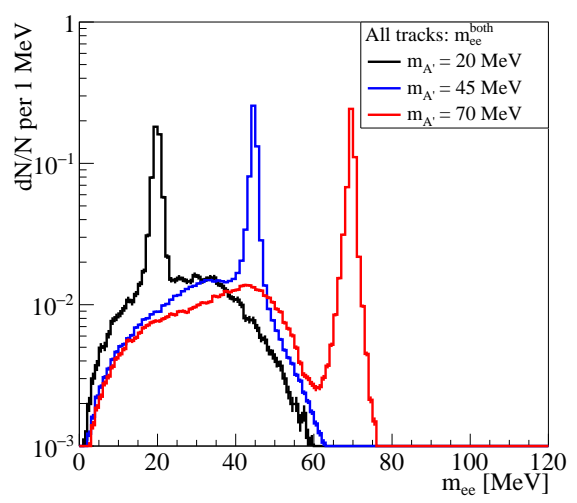

(b) Simulated dark photon signal events. Both $e^{+} e^{-}$combinations are considered.

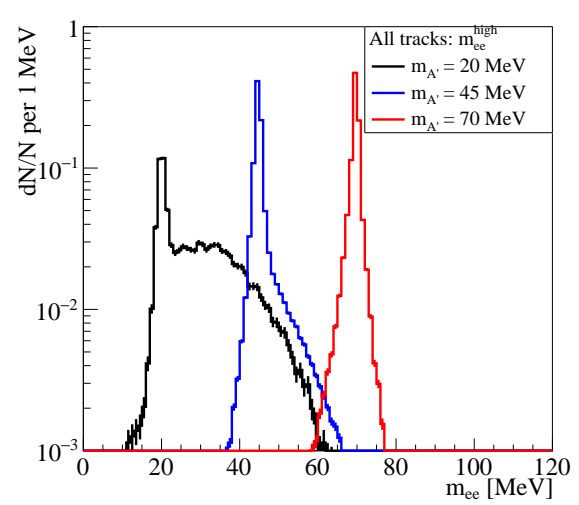

(d) Simulated dark photon signal events. The $e^{+} e^{-}$combination with the higher invariant mass is shown.

Figure 7: Distribution of the reconstructed invariant mass of $e^{+} e^{-}$pairs for simulated Standard Model background and signal from prompt dark photon decays.

decays and decay promptly to an $e^{+} e^{-}$pair given the life time is short.

The background to $e^{+} e^{-}$resonance searches is the same as for $\mu \rightarrow e e e$, but this search cannot be performed background-free as the final state is the same as in the Standard Model $\mu \rightarrow$ eee $v v$ decay.

Prompt dark photon decays in muon decays are generated with MadGraph5_aMC@NLO 2.4.3 [7] using a kinetic mixing model [8] and fed into the detector simulation. Cuts on the quality of the vertex fit and distance of the reconstructed vertex to the target are applied to reduce accidental background. Reconstructed short tracks are considered whenever the track could not be extended to a long track.

The resulting distributions of the invariant $e^{+} e^{-}$mass for signal and background are shown in figure 7. The background spectrum is smoothly declining in most of the mass range and is dominated by $\mu \rightarrow$ eee $v v$ decays. Accidental background from combinations of Bhabha scattering events and Michel decays contributes on average a factor of 800 less. Other types of accidental background are even less frequent and thus negligible.

The signal distribution shows a clear peak around the dark photon mass in addition to a broad distribution that stems from the second $e^{+} e^{-}$combination of which the positron is from the muon decay and not from the dark photon decay. The sensitivity of the dark photon search is 


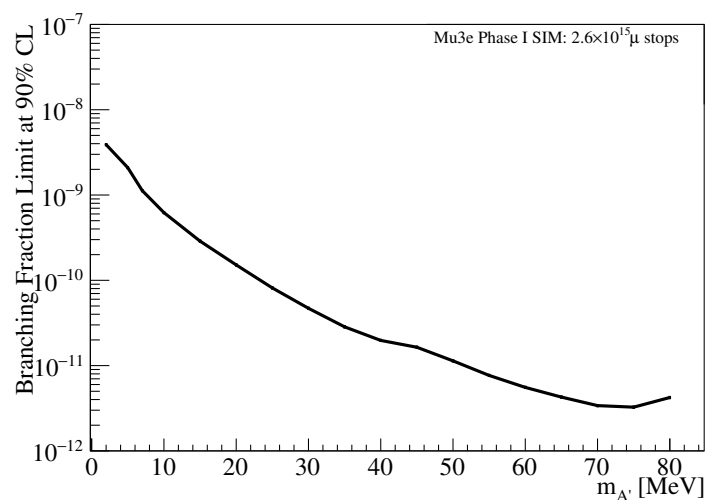

(a) Expected branching fraction limits at $90 \% \mathrm{CL}$.

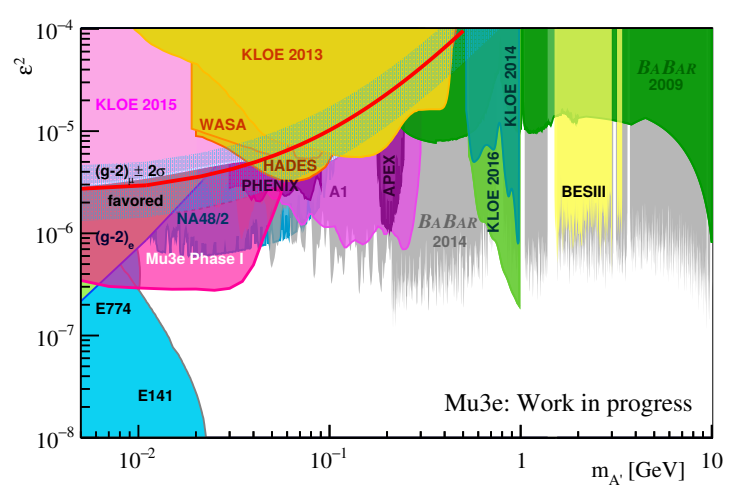

(b) Expected limits on the kinetic mixing parameter $\epsilon$ at $90 \%$ CL. Adapted from [9].

Figure 8: Sensitivity to prompt dark photon decays in $\mu \rightarrow$ eee $v v$ in the phase I Mu3e experiment.

increased by choosing the lower $m_{\mathrm{ee}}$ pair at small dark photon masses and the higher $m_{\mathrm{ee}}$ pair at high dark photon masses. The transition between the two regimes is at around $45 \mathrm{MeV}$ dark photon mass. The sensitivity is estimated in toy Monte Carlo studies. Dark photon masses $m_{A^{\prime}}$ up to $80 \mathrm{MeV}$ are studied. For higher masses, the dark photon parameter space covered by Mu3e is already excluded by existing experimental limits.

The expected limits on the branching fraction are shown in figure 8a. These range from $5 \times 10^{-9}$ at $90 \%$ CL for small dark photon masses to $3 \times 10^{-12}$ at $90 \%$ CL for high $m_{A^{\prime}}$. The branching fraction limits can be translated to limits on the kinetic mixing parameter $\epsilon$ assuming the dark photon decays exclusively to $e^{+} e^{-}$[8]. The results are shown in figure 8b. Due to the immense number of muon decays, the Mu3e experiment is capable to investigate a currently not covered parameter space.

\subsection{Lepton Flavour Violating Two-Body Decays}

A further channel that can be investigated with the Mu3e experiment is the lepton flavour violating decay $\mu \rightarrow e X$ in which $X$ denotes a neutral light particle that is not detected in the experiment. Such a decay is motivated by the familon, a potential pseudo-Goldstone boson arising from an additional broken flavour symmetry [10].

The signature of a $\mu \rightarrow e X$ decay is a monoenergetic positron whose energy is determined by the mass of $X$. It can thus be identified by a narrow peak on the smooth momentum spectrum of positrons from Standard Model muon decays.

As the filter farm only selects events with $\mu \rightarrow$ eee candidates for data storage, $\mu \rightarrow e X$ searches are performed on momentum histograms derived from online reconstruction with the drawback of the non-optimal momentum resolution of short tracks. The histograms are filled with tracks that are estimated to stem from the target and pass selections on the quality of the track fit as well as on the inclination angle. The latter selection efficiently removes tracks that stem from particles which perform multiple loops in the detector. The resulting momentum spectra for signal and background are shown in figure 9.

The mass reach for $\mu \rightarrow e X$ searches is constrained by the minimum transverse momentum of detectable positrons which allows to observe $X$ with masses of up to $95 \mathrm{MeV}$. Furthermore, the characteristic edge in the momentum spectrum of positrons from the dominant Michel decay is the preferred means of absolute momentum calibration in the baseline $\mu \rightarrow$ eee search. In this case, $\mu \rightarrow e X$ searches cannot be conducted close to the Michel edge which affects 


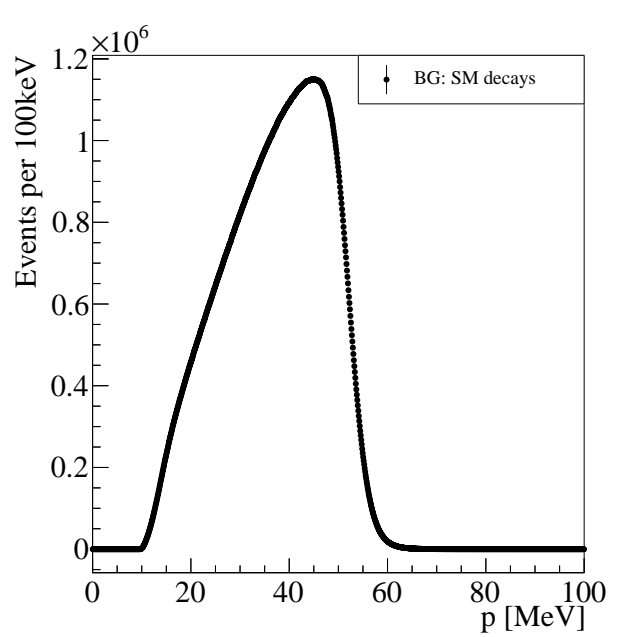

(a) Simulated background events.

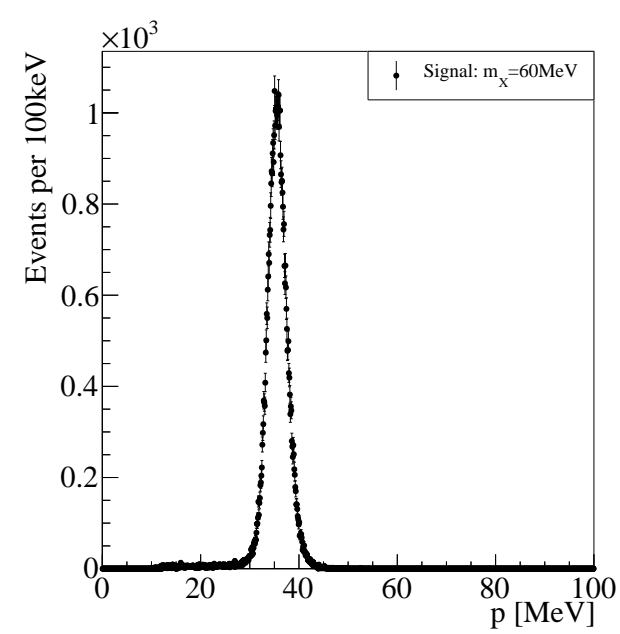

(b) Simulated $\mu \rightarrow e X$ signal events.

Figure 9: Spectra of the reconstructed momentum of positrons from simulated Standard Model background and $\mu \rightarrow e X$ signal events.

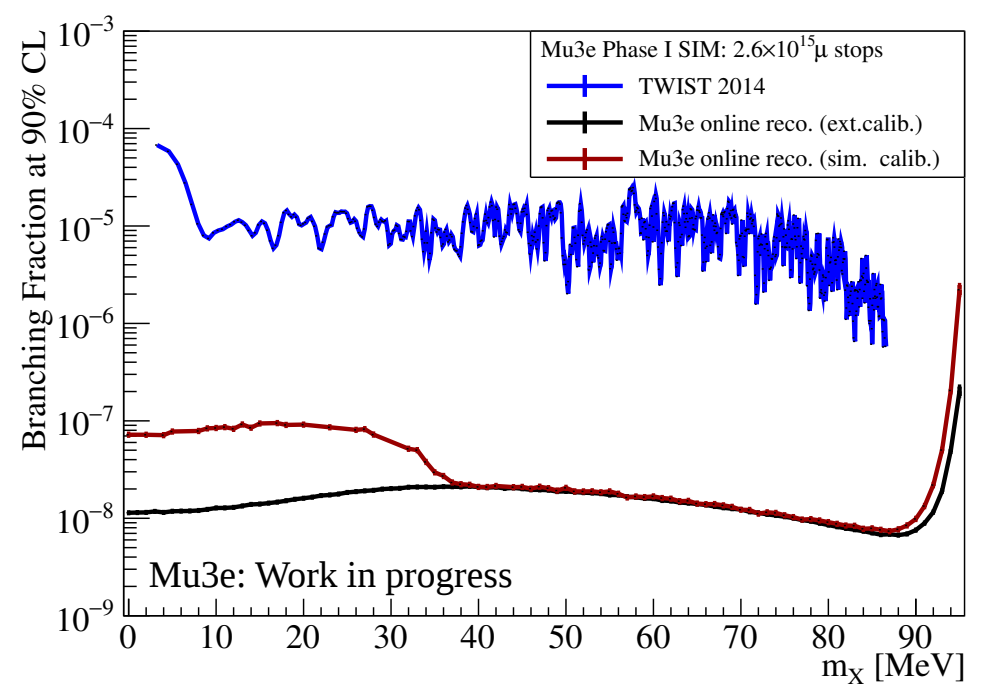

Figure 10: Expected limits at $90 \% \mathrm{CL}$ on the branching fraction of $\mu \rightarrow e X$ in the phase I Mu3e experiment. The momentum calibration is either obtained from the same momentum spectrum with a left out signal window (red line) or is assumed to be obtained from another process such as Bhabha or Mott scattering (black line). Observed limits by the TWIST experiment are shown in blue [11].

massless and light $X$. Alternative calibration methods relying on Bhabha or Mott scattering are currently being studied.

The sensitivity in the first phase of the Mu3e experiment is estimated in toy Monte Carlo studies using generated $\mu \rightarrow e X$ and Standard Model muon decays reconstructed as short tracks. For the results shown in figure 10, the momentum calibration is either assumed to stem from an alternative approach such as Bhabha scattering, or a window around the expected $\mu \rightarrow e X$ signal is left out while the calibration is performed on the Michel spectrum. In the latter case, the sensitivity deviates from the first approach as soon as the signal window reaches the Michel 
edge because the calibration becomes less precise.

The latest limits on the $\mu \rightarrow e X$ branching fraction for massive $X$ have been derived by the TWIST experiment [11]. Averaged over the mass range, branching fractions larger $9 \times 10^{-6}$ are excluded at $90 \%$ CL. Driven by the large number of muon decays, an improvement by a factor of 600 is estimated for the Mu3e experiment in phase I with expected branching fraction limits in the order of $10^{-8}$.

\section{Conclusion}

The upcoming Mu3e experiment at PSI is going to search for the lepton flavour violating decay $\mu^{+} \rightarrow e^{+} e^{-} e^{+}$with an unprecedented sensitivity. Already in phase I, a branching fraction of $5.2 \times 10^{-15}$ can be measured or excluded at $90 \%$ CL. Furthermore, conclusions on the type of underlying physics can be drawn from the decay distributions in case of discovery.

In addition to $\mu \rightarrow$ eee searches, the experiment is also suited to investigate other signatures of physics beyond the Standard Model. Substantial improvements can be expected in searches for decays of the type $\mu \rightarrow e X$ for which branching fractions in the order of $10^{-8}$ can be tested. Also in the case of dark photons a currently uncovered parameter space can be studied.

\section{Acknowledgements}

The presented work was supported by the Research Training Group 'Particle Physics Beyond the Standard Model' (GRK 1940) of the Deutsche Forschungsgemeinschaft.

\section{References}

[1] U. Bellgardt et al., Search for the decay $\mu^{+} \rightarrow e^{+} e^{+} e^{-}$, Nucl. Phys. B 299, 1 (1988), doi:10.1016/0550-3213(88)90462-2.

[2] A. Blondel et al., Research proposal for an experiment to search for the decay $\mu \rightarrow$ eee (2013), arXiv:1301.6113.

[3] I. Perić, A novel monolithic pixelated particle detector implemented in high-voltage CMOS technology, Nucl. Instrum. Methods Phys. Res. Sect. A 582, 876 (2007), doi:10.1016/j.nima.2007.07.115.

[4] N. Berger, A. Kozlinskiy, M. Kiehn and A. Schöning, A new three-dimensional track fit with multiple scattering, Nucl. Instrum. Methods Phys. Res. Sect. A 844, 135 (2017), doi:10.1016/j.nima.2016.11.012.

[5] Y. Kuno and Y. Okada, Muon decay and physics beyond the standard model, Rev. Mod. Phys. 73, 151 (2001), doi:10.1103/RevModPhys.73.151.

[6] A. Crivellin, S. Davidson, G. M. Pruna and A. Signer, Renormalisation-group improved analysis of $\mu \rightarrow e$ processes in a systematic effective-field-theory approach, J. High Energ. Phys. 05, 117 (2017), doi:10.1007/JHEP05(2017)117.

[7] J. Alwall, R. Frederix, S. Frixione, V. Hirschi, F. Maltoni, O. Mattelaer, H. S. Shao, T. Stelzer, P. Torrielli and M. Zaro, The automated computation of tree-level and next-toleading order differential cross sections, and their matching to parton shower simulations, J. High Energ. Phys. 07, 079 (2014), doi:10.1007/JHEP07(2014)079. 
[8] B. Echenard, R. Essig and Y.-M. Zhong, Projections for dark photon searches at Mu3e, J. High Energ. Phys. 01, 113 (2015), doi:10.1007/JHEP01(2015)113.

[9] M. Ablikim et al. [BESIII Collaboration], Dark photon search in the mass range between 1.5 and $3.4 \mathrm{GeV} / \mathrm{c}^{2}$, Phys. Lett. B 774, 252 (2017), doi:10.1016/j.physletb.2017.09.067.

[10] F. Wilczek, Axions and family symmetry breaking, Phys. Rev. Lett. 49, 1549 (1982), doi:10.1103/PhysRevLett.49.1549.

[11] R. Bayes et al., Search for two body muon decay signals, Phys. Rev. D 91, 052020 (2015), doi:10.1103/PhysRevD.91.052020. 